\title{
Grzegorz Malinowski
}

\section{IDENTITY, MANY-VALUEDNESS AND REFERENTIALITY}

\begin{abstract}
In the paper* we discuss a distinctive versatility of the nonFregean approach to the sentential identity. We present many-valued and referential counterparts of the systems of SCI, the sentential calculus with identity, including Suszko's logical valuation programme as applied to manyvalued logics. The similarity of different constructions: many-valued, referential and mixed, leads us to the conviction of the universality of the non-Fregean paradigm of sentential identity as distinguished from the equivalence, cf. [9].
\end{abstract}

Keywords: identity; many-valuedness; referential semantics; non-Fregean logic; sentential calculus with identity; SCI

\section{Introduction}

The classical sentential logic has a distinguished semantics provided by the two-element matrix $\mathcal{M}_{2}$. One consequence of this fact is that the equivalence connective meets the Fregean condition that two sentences having the same logical values describe the same, i.e. they have the same referent or, denotation. In other words, we may say that the only attribute of the sentence within the classical logic is its truth-value. The truth table condition for the equivalence is the following:

$$
x \leftrightarrow y \in\{1\} \text { if and only if } x=y .
$$

* The paper is an advanced and extended version of [4]. The author is grateful to the editors of the volume in which the latter appeared for their kind permission to use here some material from it. 
The right hand side equality of the formula merely serves as the equality of logical values and not the identity of sentences in any extended or a deeper sense. Accordingly, the truth tables semantics covers only a small part of the ontology of referents of sentences and in no reasonable sense describes contents of these linguistic entities.

In order to avoid the inconvenience, Suszko [9] extended the classical logic introducing in its language a non truth-functional connective of identity, denoted henceforth as $\equiv$. The intended meaning of the new connective is explained by Suszko and his collaborators in several ways, two of which are important for our purposes, namely through the use of logical valuations and models, see [9] and [1]. The latter description, by models with the designated set of elements, enables us to express the fact that two sentences are identical, modulo given model, whenever their semantic correlates are identical. The choice of the class of models determines the kind of sentential identity, that is applied to specific structures of semantic correlates or, as Suszko would say, (structures of) situations. It is crucial that every model distinguishes between two kinds of situations: the positive ones, i.e. these which obtain, and the negative ones. The weakest logic of identity SCI, the Sentential Calculus of Identity, may be characterized semantically by the use of SCI-models, see Bloom [1]. Among the extensions and theories of the SCI - the basic system of the identity connective - one may find several known and important systems of modal logic and the many-valued logics. the fact that SCI, in the class of its models has the matrices of many-valued logics and, moreover, that these logics appear to be axiomatic strengthenings of SCI gives to many-valuedness a new dimension.

The aim of the paper is to show a specific universality of the nonFregean paradigm of sentential identity as distinct from equivalence, cf. [9]. To achieve this goal, we discuss several issues from some earlier works by the author, including [3], [4], [5] and [7]. Our first task is to compare the non-Fregean interpretation of finite many-valued Łukasiewicz logics, [3], and the interpretation of the Rosser and Turquette standard logics, using a common terminology of the SCI-models, [6]. Accordingly, the finite Rosser and Turquette logics are seen as classical protolog$i c s$ based on $n$-element set of values and the identity connective expressed in terms of $n$-element conjunction of formulas indicating the equivalence of formulas modulo a given logical value. In the sequel, we present the referential semantics by Wójcicki. The latter is best characterized through the selfextensionality, which resembles the Fregean Axiom - the property 
identifies the external semantic congruence relations with the equivalence of formulas. The former bunch of relations is a counterpart of the identity in non-Fregean logic. Next, the $m$-valued referential semantics, received in result of generalization of referentiality to the case inspired by the Rosser ad Turquette construction principles. It is shown that the problem of identity in the referential framework is confined to the external congruence relation of formulas and that, in turn, this inferential equivalence expressed with the matrix consequence operation $C$ may be distributed as the intersection of the $C$-equivalence and the inferential $C$-equivalences modulo specific formulas displaying (or, imitating) logical values which, in the construction, are the reference points.

\section{Identity and its models}

The basic and the weakest system of the propositional identity, SCI, Sentential Calculus of Identity, is formulated in a standard sentential language extended with the additional, special connective of identity $\equiv$. Its semantical description in terms of SCI-models was given by Bloom in [1]. An SCI-model is a matrix $\mathcal{M}=(\mathcal{A}, D)$, founded on the algebra

$$
\mathcal{A}=(A, \neg, \rightarrow, \vee, \wedge, \leftrightarrow, \equiv)
$$

and such that for any $a, b \in A$

$$
\begin{array}{lll}
\neg a \in D & \text { if and only if } & a \notin D, \\
a \rightarrow b \notin D & \text { if and only if } & a \in D \text { and } b \notin D, \\
a \vee b \notin D & \text { if and only if } & a \notin D \text { and } b \notin D, \\
a \wedge b \in D & \text { if and only if } & a \in D \text { and } b \in D, \\
a \leftrightarrow b \in D & \text { if and only if } & a, b \in D \text { or } a, b \notin D, \\
a \equiv b \in D & \text { if and only if } & a=b .
\end{array}
$$

As it is is easily seen, all the connectives except the identity are described classically with respect to the division of the set of elements of the matrix $\mathcal{M}$ into two subsets: the set of designated elements $D$ and the set of undesignated elements $A \backslash D$. The matrix SCI consequence relation $\models_{\mathrm{SCI}}$ is defined as follows:

$$
X \models_{\mathrm{SCI}} \alpha \text { if and only if } X \models_{\mathcal{M}} \alpha \text { for any SCI-model } \mathcal{M} \text {. }
$$

The interpretations of the SCI language $\mathcal{L}=($ For, $\neg, \rightarrow, \vee, \wedge, \leftrightarrow, \equiv)$ are homomorphisms $h: \mathcal{L} \rightarrow \mathcal{A}$. Moreover, for any such $h$ there corre- 
sponds a valuation $t_{h}$ : For $\rightarrow\{0,1\}$ such that:

$$
t_{h}(\alpha)=1 \text { if and only if } h(\alpha) \in D .
$$

The valuations $t_{h}$ describe the connectives $\neg, \rightarrow, \vee, \wedge, \leftrightarrow$ in terms of truth values in precisely the same way as in the classical logic, i.e. in terms of truth tables. Contrary to this, the identity connective is described directly, in relation to homomorphisms

$$
t_{h}(\alpha \equiv \beta)=1 \text { if and only if } h(\alpha)=h(\beta) .
$$

Accordingly, SCI may be described using the binary logical valuations.

The logical valuation procedure forms a part of a broader semantical programme related to the general conception of non-Fregean logics, cf. [9]. According to Suszko there are situations which play the role of semantic correlates of propositions. Logical valuations, for their part, are nothing more than characteristic functions of the sets of formulae that are counterimages of the sets of positive situations, i.e. of those which obtain, under homomorphisms settling the interpretation.

In case of pure SCI, neither the quantity nor the quality of situations are established. Besides, that is why the number of the strengthenings of this calculus and their expressing power is only limited by the large class of its models. Accordingly, the class of the already known strengthenings of SCI include the highly recognized systems of modal logic, where the connectives of necessity and possibility are defined using the identity in the models fulfilling Boolean and some other properties, cf. [9].

The two-element classical matrix $\mathcal{M}_{2}=(\{0,1\}, \neg, \rightarrow, \vee, \wedge, \leftrightarrow, \equiv$, $\{1\})$ in which the equivalence $\leftrightarrow$ and the identity $\equiv$ coincide is the extreme SCI model. Obviously, the model $\mathcal{M}_{2}$ is Fregean - it is based on two situations, 0 and 1, which stand for the logical values of falsity and truth. This naturally suggests that considering many-valued logics in non-Fregean framework might be of special interest.

With Suszko there originated an approach to many-valued logics in terms of logical valuations, i.e. the description using bi-valent logical valuations as opposed to algebraic reference assignments. In [10] he provided a bi-valent description for the three-valued Łukasiewicz sentential calculus, which may also be treated as the first step towards thinking about many-valued logics as axiomatic strengthenings of SCI. The author of the present paper showed in [3] that every finite $n$-valued Eukasiewicz logic has a bivalent description and, thus, it is logically twovalued. And, referring to appropriate tautological many-valued equiv- 
alences, he also proved that all finite Łukasiewicz logics are axiomatic strengthenings of SCI.

In the next section, we present the $n$-valued Łukasiewicz logic ( $n$ finite) as a two-valued logic of $n$ situations denoted by Eukasiewicz 0 , $1 / n-1,2 / n-1, \ldots, 1$ original values. We also discuss the second quality of Łukasiewicz systems.

\section{Lukasiewicz SCI-models}

Consider a finite $n$ element set $E_{n}=\left\{0,{ }^{1} /{ }_{n-1},{ }^{2} /{ }_{n-1}, \ldots, 1\right\}, n \geqslant 2$, of logical values on which there are defined the connectives of negation $(\sim)$, implication $(\Rightarrow)$ of Łukasiewicz: for any $x, y \in E_{n}$

$$
\begin{aligned}
\sim x & :=1-x \\
x \Rightarrow y & :=\min \{1,1-x+y\},
\end{aligned}
$$

and the connectives of disjunction $(\vee)$, conjunction $(\wedge)$ and equivalence $(\equiv)$ :

$$
\begin{aligned}
x \vee y & :=(x \Rightarrow y) \Rightarrow y=\max (x, y), \\
x \wedge y & :=\sim(\sim x \vee \sim y)=\min (x, y), \\
x \equiv y & :=(x \Rightarrow y) \wedge(y \Rightarrow x)=\min (1,1-|x-y|) .
\end{aligned}
$$

Using the original Łukasiewicz's negation and implication one may additionally define the connectives $\neg, \rightarrow$ :

$$
\begin{aligned}
\neg x & :=x \Rightarrow_{n-1} \sim(x \equiv x), \\
x \rightarrow y & :=x \Rightarrow_{n-1} y,
\end{aligned}
$$

behaving classically with respect to the division of $E_{n}$, when 1 is the only designated element: ${ }^{1}$

$$
\begin{gathered}
\neg x=1 \text { if and only if } x \neq 1, \\
x \rightarrow y=1 \text { if and only if } x \neq 1 \text { or } y=1 .
\end{gathered}
$$

In that case $\equiv$ is the identity connective,

$$
x \equiv y=1 \text { if and only if } x=y,
$$

in the SCI-model $\mathcal{A}_{n}=\left(E_{n}, \neg, \rightarrow, \vee, \wedge, \leftrightarrow, \equiv,\{1\}\right)$, cf. [3].

${ }^{1} x \Rightarrow_{n-1} y$ is an "ascending" implication $\left.\alpha \rightarrow(\alpha \rightarrow \ldots \rightarrow(\alpha \rightarrow \beta) \ldots)\right)$ with $(n-1)$ antecedents. The function is described by the formula $\min (1,(n-1)(1-x)+y)$. 
The SCI models generated by finite Eukasiewicz matrices have this particular property that the original Łukasiewicz connectives of negation and implication are also definable. What is more, the definitions of these connectives refer to the identity and the classical negation, conjunction and/or disjunction, and the following logical laws: ${ }^{2}$

$$
\begin{aligned}
(\sim \alpha) & \equiv(\alpha \equiv \neg(\alpha \equiv \alpha)), \\
(\alpha \rightarrow \beta) & \equiv((\alpha \wedge \beta) \equiv \alpha), \\
(\alpha \rightarrow \beta) & \equiv((\alpha \vee \beta) \equiv \beta) .
\end{aligned}
$$

Bloom and Brown [2] shifted the classical properties of the connectives onto the properties of consequence. They studied finite consequence operations that have the properties

$(\neg) \quad C(X, \neg \alpha)=$ For if and only if $\alpha \in C(X)$,

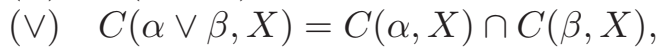

characteristic for the classical logic and for SCI and called them classical. Bloom and Brown also showed that any such consequence $C$ is an axiomatic strengthening of the consequence of $C_{2}$, i.e. it is the consequence of the classical logic. In any single case there exists a set of formulas (axioms) $Y \subseteq$ For such that $C(X)=C_{2}(X \cup Y)$.

Now, notice that the following formulas

$$
\begin{aligned}
(\alpha \vee \beta) & \equiv((\alpha \Rightarrow \beta) \Rightarrow \beta), \\
(\alpha \wedge \beta) & \equiv(\sim(\sim \alpha \vee \sim \beta)), \\
\neg \alpha & \equiv\left(\alpha \Rightarrow_{n-1} \sim(\alpha \equiv \alpha)\right), \\
(\alpha \rightarrow \beta) & \equiv(\neg \alpha \vee \beta), \\
(\alpha \equiv \beta) & \equiv((\alpha \Rightarrow \beta) \wedge(\beta \Rightarrow \alpha)) .
\end{aligned}
$$

belong to $C_{n}(\emptyset)$, where $C_{n}$ is the consequence of Łukasiewicz $n$-valued logic defined on the extended language containing the additional classical connectives of negation $\neg$ and implication $\rightarrow$.

Hence any finite Eukasiewicz logic is classical in the above sense, cf. [2], and it is an axiomatic strengthening of the classical logic. Let us also recall that, due to Suszko's thesis, each of them considered as a matrix consequence is logically two-valued and may be described in terms of $0-1$ valuations, see [3] and [9].

\footnotetext{
${ }^{2}$ In [9] R. Suszko refers to them as to the laws of the three-valued logic.
} 


\section{Rosser and Turquette protologics}

Given a finite $n$ element set $E_{n}=\left\{0,1 / n-1,{ }^{2} / n-1, \ldots, 1\right\}, n \geqslant 2$, consider any $k$, such that $1<k \leqslant n-1$, and the following set of designated values:

$$
D_{k}=\{n-k+1 / n-1, \ldots, 1\} \text {. }
$$

The many-valued Rosser-Turquette logics [8] are defined on the matrices of the form

$$
\mathcal{M}_{n, k}=\left(\mathcal{U}_{n}, D_{k}\right),
$$

where $\mathcal{U}_{n}=\left(E_{n}, f_{1}, f_{2}, \ldots, f_{m}\right) .^{3}$

The core idea of the author's approach consists in considering the socalled standard connectives having the properties similar to the properties of their classical logic counterparts and some extra connectives important from other point of view. So, the functions $\neg, \rightarrow, \vee, \wedge, \leftrightarrow$ of the matrix $\mathcal{M}_{n, k}$ corresponding to negation, implication, disjunction, conjunction and the equivalence for any $x, y \in E_{n}$ fulfill, respectively, the following conditions

$$
\begin{array}{lll}
\neg x \in D_{k} & \text { if and only if } & x \notin D_{k} \\
x \rightarrow y \notin D_{k} & \text { if and only if } & x \in D_{k} \text { and } y \notin D_{k} \\
x \vee y \notin D_{k} & \text { if and only if } & x \notin D_{k} \text { and } y \notin D_{k} \\
x \wedge y \in D_{k} & \text { if and only if } & x \in D_{k} \text { and } y \in D_{k} \\
x \leftrightarrow y \in D_{k} & \text { if and only if } & x, y \in D_{k} \text { or } x, y \notin D_{k} .
\end{array}
$$

Notice that any matrix

$$
\mathcal{K}_{n, k}=\left(E_{n}, \neg, \rightarrow, \vee, \wedge, \leftrightarrow, D_{k}\right)
$$

is epimorphic to the classical matrix $\mathcal{M}_{2}=(\{0,1\}, \neg, \rightarrow, \vee, \wedge, \leftrightarrow,\{1\})$. The mapping $e: E_{n} \rightarrow\{0,1\}$ :

$$
e(x)=\left\{\begin{array}{lll}
1 & \text { if } & x \in D_{k} \\
0 & \text { if } & x \notin D_{k}
\end{array}\right.
$$

establishes the epimorphism.

Rosser and Turquette, in their $n$-valued logics, also admit one-argument connectives $J=\left\{j_{0}, j_{1}, \ldots, j_{n-1}\right\}$, such that their matrix counterparts are "characteristic functions" of logical values:

$$
j_{i}(x) \in D_{k} \text { if and only if } x=i / n-1
$$

3 Rosser and Turquette originally use $1,2, \ldots, k, \ldots, n$ for marking the elements of the matrix. 
are definable. It appears that any standard matrix $\mathcal{M}_{n, k}$ equipped with $j$ 's is a good candidate for an SCI model. Actually, $\mathcal{M}_{n, k}$ may be extended to such a model under the condition of definability of the identity connective in its algebraic structure. This is just the case, since putting

$$
x \equiv y=\left(j_{0}(x) \leftrightarrow j_{0}(y)\right) \wedge\left(j_{1}(x) \leftrightarrow j_{1}(y)\right) \wedge \cdots \wedge\left(j_{n-1}(x) \leftrightarrow j_{n-1}(y)\right)
$$

and using the properties of the connectives on the right side one may easily check that for any interpretation $h$ of the language $L$ in the matrix $\mathcal{M}_{n, k}$, i.e. for any homomorphism $h: L \rightarrow U_{n}$,

$$
h(\alpha \equiv \beta) \in D_{k} \text { if and only if } h(\alpha)=h(\beta) .
$$

Thus, $\equiv$ is the sentential identity in the standard logic and the matrix

$$
\mathcal{M}_{n, k}=\left(E_{n}, \neg, \rightarrow, \vee, \wedge, \leftrightarrow, \equiv, D_{k}\right)
$$

is an SCI-model.

In the given SCI model the identity may be represented as the conjunction of the equivalences $\equiv_{i}$ related to the logical correlates in the following way:

$$
\alpha \equiv_{i} \beta=j_{i}(\beta) \leftrightarrow j_{i}(\beta) .
$$

We then get that the identity of formulas in the standard Rosser and Turquette logic splits on the conjunction of $n$ equivalences corresponding to the logical values:

$$
\alpha \equiv \beta=\left(\alpha \equiv_{0} \beta\right) \wedge\left(\alpha \equiv_{1} \beta\right) \wedge \cdots \wedge\left(\alpha \equiv_{n-2} \beta\right) \wedge\left(\alpha \equiv_{n-1} \beta\right) .
$$

Let us remark, in the end, that standard Rosser and Turquette logics meet Bloom and Brown [2] conditions. Any consequence operation $C_{n, k}$ corresponding to the relation of consequence $\models_{n, k}$ of the matrix $\mathcal{M}_{n, k}$ :

$$
\alpha \in C_{n, k}(X) \text { if and only if } X \models_{n, k} \alpha
$$

is, therefore, an axiomatic extension of $C_{2}: C_{n, k}(X)=C_{2}(X \cup Y)$ for some set(of axiom schemes) $Y \subseteq$ For. Finally, every logic $\left(L, C_{n, k}\right)$ delineates a referential logic of $n$ situations in which the following formulas are tautologies:

$$
\begin{gathered}
j_{0}(p) \vee j_{1}(p) \vee \ldots \vee j_{n-1}(p), \\
\neg\left(j_{s}(p) \wedge j_{t}(p)\right) \text { for } s, t \in\{1, \ldots, n-1\} .
\end{gathered}
$$

The conclusion is that these minimal models of the logic with identity define the weakest logics of $n$-element identity and, similarly to SCI, large 
classes of strenghtenings. The investigation of these classes seems to be interesting and it may result in important settlements on the class of the strengthenings of SCI.

\section{Referential semantics and identity}

The referential semantics is based on the assumption that the logical value of a sentence may depend on additional conditions treated as the reference points. The methodology of referential matrices for structural consequence operations was proposed and elaborated by R. Wójcicki in [11]. The central theorem concerning the scope of this kind of semantics leads to the reflection on the similarity between the congruence of some kind of structural consequence and the sentential identity. On the other hand, the generalization of the referential semantics onto a many-valued case in [5] (see also [7]) makes the problem of expressing of the identity similar to the description in SCI models based on standard Rosser and Turquette matrices - we deal with the problem more thoroughly in Section 5.

A referential algebra is any algebra $\mathcal{A}=\left(A, f_{1}, f_{2}, \ldots, f_{n}\right)$, with $A$ being a subset of $\{0,1\}^{T}$, i.e., the elements of $A$ are mappings of $T$ into $\{0,1\}$. For any $t \in T$, the symbol $D_{t}$ denotes the set of referential functions $r \in A$, which on $t$ take the value $1, D_{t}:=\{r \in A: r(t)=1\}$. Then

$$
\mathcal{W}=\left(\mathcal{A},\left\{D_{t}, t \in T\right\}\right)
$$

is a referential matrix, cf. [11].

For any referential matrix, based on the algebra similar to a sentential language $\mathcal{L}$, the structural consequence $C n_{\mathcal{W}}$ defined by the valuations $h: \mathcal{L} \rightarrow \mathcal{A}$ may be defined. For any formula $\alpha$ and a set of formulas $X$,

$\alpha \in C n_{\mathcal{W}}(X)$ if and only if for any $h, t \in T$,

$$
(h \alpha)(t)=1 \text { whenever }(h \beta)(t)=1 \text { for all } \beta \in X .
$$

The consequence operation $C: 2^{\text {For }} \rightarrow 2^{\text {For }}$ of an arbitrary sentential language $\mathcal{L}=\left(\right.$ For $\left., F_{1}, \ldots, F_{m}\right)$, i.e. an algebra of formulas freely generated on the countable set of sentential variables, $\operatorname{Var}=\{p, q, r, \ldots\}$ by the connectives $F_{1}, \ldots, F_{m}$, is defined by the conditions:

$$
X \subseteq C(X)
$$




$$
\begin{gathered}
C(X) \subseteq C(Y), \text { if } X \subseteq Y, \\
C C(X) \subseteq C(X) .
\end{gathered}
$$

$C$ is structural provided that for any substitution $e, e C(X) \subseteq C(e X)$.

For an arbitrary structural consequence $C$ on $\mathcal{L}$ one may define two relations $\sim_{C}$ and $\approx_{C}$ puting:

$$
\begin{array}{ll}
\alpha \sim_{C} \beta & \text { if and only if } C(\alpha)=C(\beta), \\
\alpha \approx_{C} \beta & \text { if and only if } C(\varphi[\alpha / p])=C(\varphi[\beta / p]),
\end{array}
$$

for any $\alpha, \beta, \varphi \in$ For and $p \in$ Var. The first, $\sim_{C}$, is an equivalence and will be further called the $C$-equivalence. The second relation, $\approx_{C}$, is the biggest congruence $\theta$ on $\mathcal{L}$ such that

$$
\alpha \approx_{C} \beta(\theta) \text { implies } \alpha \approx_{C} \beta .
$$

For any $\alpha, \beta \in$ For $\alpha \approx_{C} \beta$ implies that $\alpha \sim_{C} \beta$. The strong completeness theorem for a structural sentential $\operatorname{logic}(\mathcal{L}, C)$ states that $C$ is complete with respect to a referential matrix $\mathcal{W}, C=C n_{\mathcal{W}}$, always, when the converse of the last implication also holds true. Any logic fulfilling this condition, i.e. $\alpha \approx_{C} \beta$ if and only if $\alpha \sim_{C} \beta$, is called selfextensional, see [11].

Selfextensionality identifies two relations: $C$-congruence and $C$ equivalence, $\approx_{C}=\sim_{C}$. This naturally suggests an analogy to the case of the identity and equivalence connectives in the classical logic, and by that to the Fregean Axiom, [9]. In other cases, for non-Fregean logics, the two relations are different; we faced it while dealing with the Eukasiewicz logics and the Rosser and Turquette protologics. In the next section we touch upon many-valuedness within the referential framework.

\section{Many-valued referential matrices}

The sentential identity and the equivalence, understood as a "classical" connective in a non-classical logics are different. Nevertheless, as we show it for standard Rosser and Turquette matrix SCI models, the identity connective may be expressed as the multiple conjunction of equivalences with respect to sharing the same value from the set $E_{n}=$ $\left\{0,1 / n-1,{ }^{2} / n-1, \ldots, 1\right\}$.

In what follows, we show a similar property for non-selfextensional logics formalized by means of special $n$-valued referential matrices inspired by the "standard" Rosser and Turquette construction, see [7]. 
Given a language $\mathcal{L}=\left(\right.$ For $\left., F_{1}, \ldots, F_{m}\right)$ and a non-empty set of reference points $T$, the ramified matrix $\mathcal{W}=(\mathcal{A}, D)$ is an $n$-valued referential matrix for $\mathcal{L}, n \geqslant 2$, if the following conditions are satisfied:

(W1) The algebra $\mathcal{A}$ is similar to $\mathcal{L}$ and the set $A$ of its elements is a subset of $\left\{e_{0}, e_{1}, \ldots, e_{n-1}\right\}^{T}$, i.e., the elements of the algebra are functions of the form $r: T \rightarrow\left\{e_{0}, e_{1}, \ldots, e_{n-1}\right\}$. Assume, for the convenience, that $e_{0}=0$ and $e_{n-1}=1$.

(W2) $D$ is a family of all sets $D_{t}:=\{r \in A: r(t)=1\}$, for $t \in T$.

(W3) If $n \geqslant 3$, then the functions $E_{1}^{0}, \ldots, E_{n-2}^{0}: A \rightarrow A$ such that

$$
E_{i}^{0}(r)(t)= \begin{cases}1 & \text { if } r(t)=e_{i} \\ 0 & \text { otherwise }\end{cases}
$$

are definable in $\mathcal{A}$.

It is obvious that the two-valued referential matrices are a special case of the many-valued matrices and they are defined only by (W1) for $n=2$ and by (W2). Any set $\boldsymbol{K}$ of $n$-valued referential matrices for $\mathcal{L}$, $n \geqslant 2$, forms an $n$-valued referential semantics. Accordingly, the logics $\left(\mathcal{L}, C n_{\boldsymbol{K}}\right)$ may be called $n$-referential.

In many-valued referentiality just introduced, it is possible to bring in the counterpart of selextensionality. This property will be referred to as a property of $n$-normality. The strong completeness theorem says then that a structural sentential $\operatorname{logic}(\mathcal{L}, C)$ is complete with respect to an $n$-valued referential matrix $\mathcal{W}, C=C n_{\mathcal{W}}$, is $n$-normal and its $C$ congruence is expressible through an intersection of $C$-equivalence and the equivalences corresponding to $n-2$ logical values. In the sequel, we shall give more precise formulation of the completeness property.

We will say that a sentential $\operatorname{logic}(\mathcal{L}, C)$ is $n$-normal, for a finite $n \geqslant 3$, if in the language $\mathcal{L}$ there are definable unary connectives $E_{1}$, $\ldots, E_{n-2}$ such that for $i, j \in\{1, \ldots, n-2\}$

$\left(\mathrm{N}_{0}\right) \quad C\left(E_{i}(p)\right) \neq$ For, $\left(\mathrm{N}_{1}\right) \quad C\left(\alpha, E_{i}(\alpha)\right)=$ For, $\left(\mathrm{N}_{2}\right) \quad C\left(E_{i}\left(E_{j}(\alpha)\right)\right)=$ For, $\left(\mathrm{N}_{3}\right) \quad C\left(E_{i}(\alpha), E_{j}(\alpha)\right)=$ For, provided that $i \neq j$,

for any $p \in \operatorname{Var}(\mathcal{L})$ and $\alpha \in$ For.

If $(\mathcal{L}, C)$ is $n$-normal, we define, for any $i \in\{1, \ldots, n-2\}$, two argument relation $\sim_{i}$ putting

$$
\alpha \sim_{i} \beta \text { if and only if } C\left(E_{i}(\alpha)\right)=C\left(E_{i}(\beta)\right) .
$$


The final, precise formulation of the completeness theorem is the following: $(\mathcal{L}, C)$ is $n$-referential if and only if it is $n$-normal and

$$
\approx_{C}=\sim_{1} \cap \cdots \cap \sim_{n-2} \cap \sim_{C}
$$

Further going similarity to the representation in Rosser and Turquette logics may be also obtained. To this aim the referential semantics receives two additional functions $E_{0}^{0}$ and $E_{n-1}^{0}$ corresponding to the reference points $e_{0}, e_{n-1}$. Then, $\sim_{C}=\sim_{n-1}$ and the $C$-congruence playing the role of the inferential identity is presented in the following way:

$$
\approx_{C}=\sim_{0} \cap \sim_{1} \cap \cdots \cap \sim_{n-2} \cap \sim_{n-1} .
$$

Comparing this equality to the formula used for description of the identity connective in $n$-valued Rosser and Turquette logic, we find the same structural expressibility of the identities located on different semantical levels.

\section{Final remarks}

Rosser and Turquette determined the conditions that make finitelyvalued sentential logics resemble more the classical logic, and hence simplified the problem of axiomatization. We remarked that in any standard $n$-valued logic it is also possible to define the identity connective and by this, its matrix may be redefined as an SCI model. In turn, the identity may be represented as an intersection of $n$ equivalence relations corresponding to the original values. Accordingly, every minimal model of the logic with identity define precisely some weakest logic of $n$-element identity.

Our interest in Wójcicki's referential matrices was forced by the identity on the inferential grounds. It it straightforward that the selfextensionality condition of the existence of the referential matrix, and by that a referential semantics, resembles very much the Fregean postulate. A logic $(\mathcal{L}, C)$ is selfextensional whenever two inferential relations: $C$-congruence and $C$-equivalence coincide. So, we decided to match the referentiality with Rosser and Turquette many-valuedness. The resulting many-valued referential semantics is non-Fregean and its identity may also be represented as an intersection of $n$ equivalences corresponding to the original values. 
The similarity of these constructions, expressed by the decomposition of the identity on the interesection of the equivalences shows that the descriptions and criteria of identity may be formulated on several levels of the logical "engagement".

\section{References}

[1] Bloom, S. L., "A completeness theorem for 'theories of kind W'", Studia Logica 27 (1971): 43-55. DOI: 10.1007/BF02282544

[2] Bloom, S. L., and D. J. Brown, "Classical abstract logics", Dissertationes Matemathicae CII (1973): 43-52.

[3] Malinowski, G., "Classical characterization of $n$-valued Łukasiewicz calculi", Reports on Mathematical Logic 9 (1977): 41-43.

[4] Malinowski, G., "Identyczność i wielowartościowość", pages 107-115 in Identyczność znaku czy znak identyczności?, J. Golińska-Pilarek and A. Wójtowicz (eds.), Wydawnictwa Uniwersytetu Warszawskiego, Warszawa, 2012.

[5] Malinowski, G., "Many-valued referential matrices", Bulletin of the Section of Logic 24/3 (1995): 140-146.

[6] Malinowski, G., "Modes of many-valuedness", pages 159-198 in Truth in Perspective. Recent Issues in Logic, Representation and Ontology, C. Martinez, U. Rivas and L. Villegas-Forero (eds.), Ashgate, 1998.

[7] Malinowski, G., "Referentiality and matrix semantics", Studia Logica 97, 2 (2011): 297-312. DOI: 10.1007/s11225-011-9307-5

[8] Rosser, J. B., and A.R. Turquette, Many-valued Logics, North-Holland, Amsterdam, 1952.

[9] Suszko, R., "Abolition of the Fregean axiom", pages 169-239 in: Logic Colloquium. Symposium on Logic held at Boston, R. Parikh (ed.), Lecture Notes in Mathematics, vol. 453, 1972. DOI: 10.1007/BFb0064874

[10] Suszko, R., "Remarks on Eukasiewicz's three-valued logic", Bulletin of the Section of Logic 4, 3 (1975): 87-90.

[11] Wójcicki, R., Theory of Logical Calculi. Basic Theory of Consequence Operations, Synthese Library, 199, Kluwer Academic Publishers, Dordrecht, 1988.

Grzegorz MaLinowski

Department of Logic

University of Łódź

Łódź, Poland

gregmal@uni.lodz.pl 